\title{
Reducing Institutional Imaging Costs for Stereotactic Brain Biopsy
}

\author{
Francia Y FANG, BS ${ }^{1}$, I Jonathan POMERANIEC, MD, MBA ${ }^{2}$, Panagiotis MASTORAKOS, \\ $M D, P h D^{2}$ and John D HEISS, MD ${ }^{2 *}$
}

${ }^{1}$ University of Maryland School of Medicine, USA

${ }^{2}$ National Institutes of Health, National Institutes of Neurological Disorders and Stroke, Surgical Neurology Branch, USA

\begin{abstract}
Background: Imaging workup preceding stereotactic brain biopsy differs among institutions. The novel coronavirus disease-2019 (COVID-19) pandemic has prompted reallocation of scarce medical resources and reduction of unnecessary procedures. The relevant costs and value to the health system of multiple magnetic resonance imaging (MRI) scans during this workup remain poorly defined. In the current review, we specifically delineate and compare the relevant costs and value to the health system of multiple MRI scans during workup for stereotactic brain biopsy.
\end{abstract}

Methods: A generalized total cost equation was formulated and used to analyze differential cost drivers across different patient scenarios from diagnosis to surgery.

Results: Each additional MRI scan performed between diagnostic MRI and stereotactic brain biopsy contributes $\$ 306$ for Medicare patients versus $\$ 870-\$ 990$ for patients with private insurance. The main driver for imaging costs before stereotactic brain biopsy is the number of MRI scans performed between diagnosis and surgery. Imaging costs vary significantly among insurance types.

\begin{abstract}
Conclusion: There is little to no clinical value of repeating additional MRI scans if outside institution MRI scans are highquality and can be sourced to the intraoperative neuronavigation system. Cost and waste reduction is achieved through reducing the number of MRI scans by using high-quality outside institution MRI scans, minimizing the interval between scanning and surgery, avoiding dedicated MRI scans with skin fiducials for neuronavigation, and not requiring another diagnostic MRI scan from within the treating institution.
\end{abstract}

Keywords

Stereotactic biopsy, Magnetic resonance imaging, Cost, Insurance

\section{Introduction}

Stereotactic brain biopsy is commonly performed to obtain brain tissue for histopathologic and molecular analysis. Diagnostic accuracy is high and complication rates are low [1]. Frameless neuronavigation systems align MRI spatial data with head and brain anatomy in the operating room using either: 1) Contrast-enhancing scalp markers (fiducials) seen on the MRI and scalp; or 2) Scalp contour seen on an MRI model and on a scalp surface model created by tracing the immobilized scalp surface. This alignment registers the brain and MRI coordinate spaces during surgery [2]. Images on the neuronavigation system are used to plan a safe trajectory to target the tumor, avoiding vital adjacent structures and blood vessels [3]. The biopsy needle is advanced through the brain and into the lesion. Deep lesions require especially precise trajectories because angular error produces greater spatial offset with increasing depth [4].

Some neurosurgeons prefer scalp fiducial markers over scalp surface tracing for registering the MRI and head surface anatomy in the operating room. Digitized mapping of the scalp surface can supplement fiducial marker registration [5], but may not significantly improve real-time accuracy. Scalp surface-based neuronavigation can be performed using any recent high-resolution MRI scan, whereas fiducial markerbased neuronavigation requires a dedicated MRI scan with fiducials in place [2]. Clinical MRI scans may be obtained at the

*Corresponding author: John D Heiss, MD, National Institutes of Health, National Institutes of Neurological Disorders and Stroke, Surgical Neurology Branch, Bethesda, 20892, USA

Accepted: February 10, 2022

Published online: February 12, 2022

Citation: FANG FY, POMERANIEC IJ, MASTORAKOS P, et al. (2022) Reducing Institutional Imaging Costs for Stereotactic Brain Biopsy. J Neurosurg Res Rev 5(1):76-83 
surgical institution for scalp surface-based neuronavigation despite the availability of recent high-resolution brain MRI scans suitable for surgical planning from another institution [6].

The process of image acquisition and effective planning before lesional biopsy is well documented [7-10]. However, the number and types of MRI scans performed between initial diagnosis and biopsy varies among neurosurgeons based on practice preferences, number of treating institutions, patient insurance networks, and institutional affiliations. Suspected pathology and time interval between scanning and surgery are additional factors [11-13].

In current neurosurgery practice, there are several ways of planning for a stereotactic brain biopsy: One MRI scan from an outside institution (the diagnostic scan); both an outside MRI scan and a repeated MRI scan at the surgical institution; finally, multiple MRI scans at the surgical institution alone. During the novel COVID-19 pandemic when medical resources were limited, unnecessary procedures were especially discouraged. Recently, at the National Institutes of Health (NIH), we performed a stereotactic brain biopsy for a patient with relapsed primary lymphoma using a previous brain MRI scan from an outside hospital. Intraoperative neuronavigation based on this outside MRI imaging data was used to guide implantation of a right frontal Ommaya reservoir and diagnostic stereotactic biopsy of the right parietal lesion. The patient tolerated the procedure well, which prompted the authors to investigate the differential cost drivers and clinical value derived from multiple imaging studies.

\section{Methods}

The purpose of this analysis was to compare different clinical scenarios of preoperative imaging workup for stereotactic brain biopsy and understand the underlying cost drivers. Cost variables included brain MRI scans with and without contrast, brain MRI scans with contrast, type of insurance, method of anatomic registration (fiducial markers or scalp tracing), and whether brain MRI scans required a transfer from an outside institution. These costs were determined based on institutional practice patterns and calculated using public and private cost data. For the different clinical scenarios, we defined each step of the process between diagnosis and surgery. Cost data for each step was then identified and included in a generalized cost formula to determine the overall costs for each scenario (Supplementary

\section{A 2 MRIs, fiducial markers}

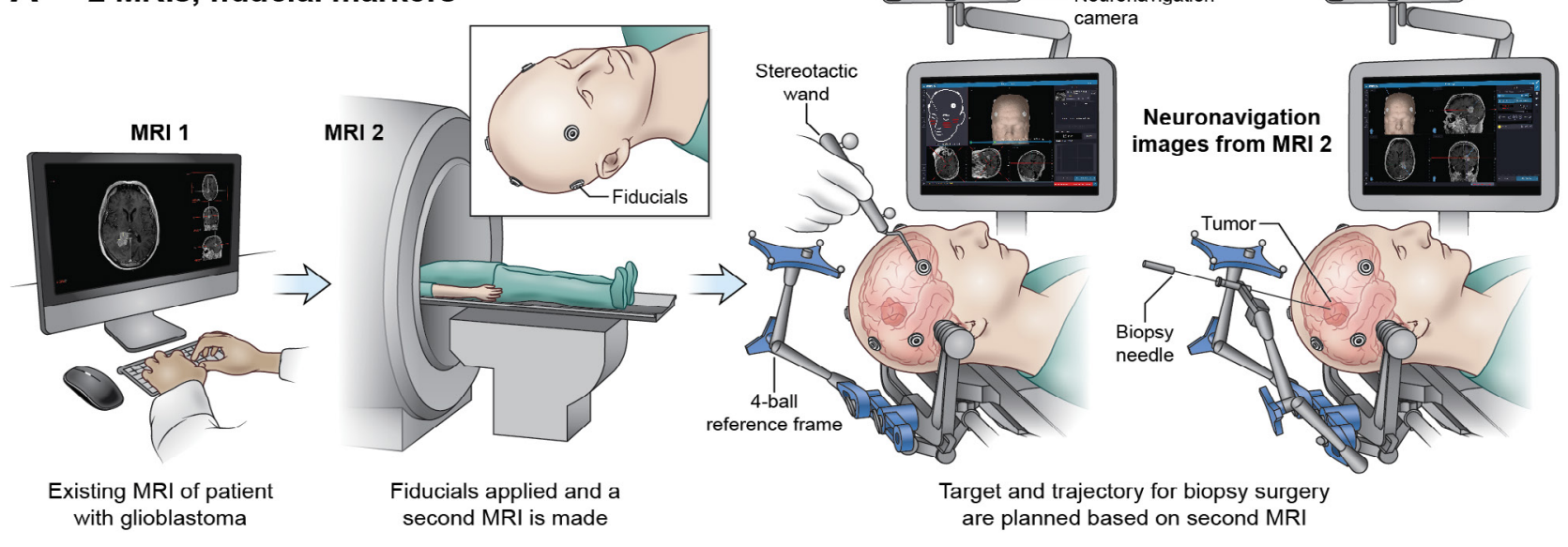

\section{B 1 MRI, no fiducial markers}
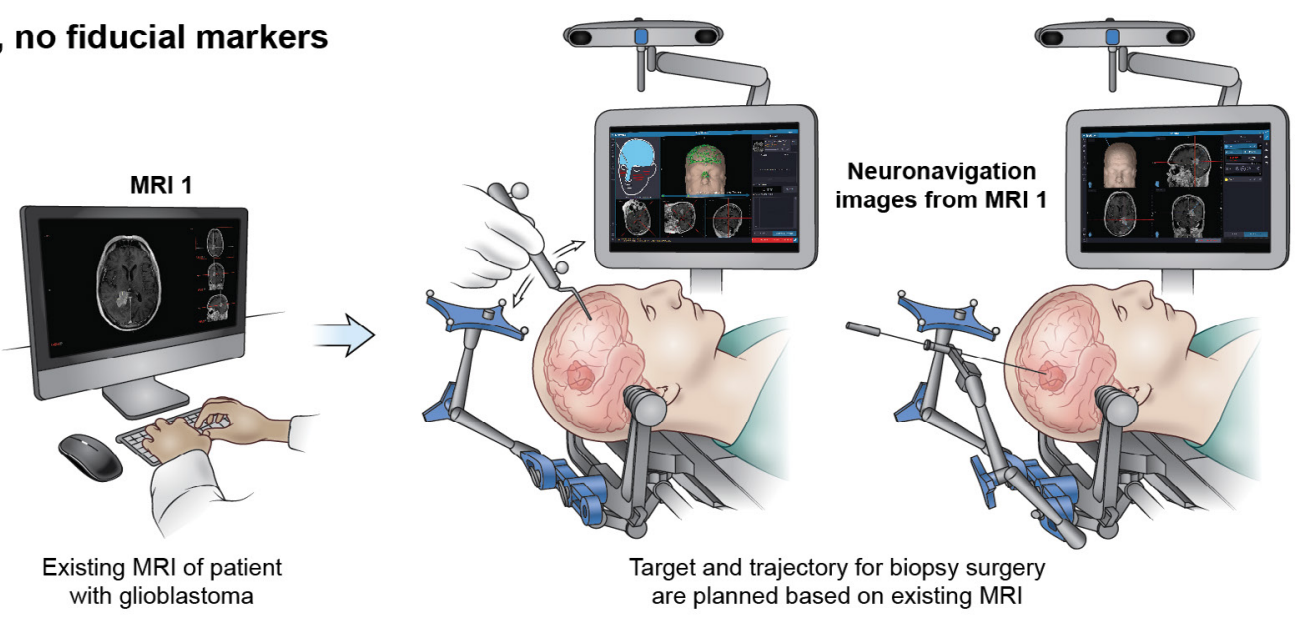

Figure 1: (A) Illustration detailing the process of applying fiducial markers or employing scalp tracing (B) to register the patient's anatomy with the neuronavigation system. 
Citation: FANG FY, POMERANIEC IJ, MASTORAKOS P, et al. (2022) Reducing Institutional Imaging Costs for Stereotactic Brain Biopsy. J Neurosurg Res Rev 5(1):76-83

Methods). We did not compare costs relative to patient outcomes.

\section{Results}

Patients without outside MRI scans typically undergo repeat imaging with or without fiducial markers before surgery (Figure 1). Standard practice and imaging workup for patients undergoing stereotactic brain biopsy at the NIH includes a high-resolution MRI scan before surgery (Figure 2). Factors influencing total cost include number of MRI scans

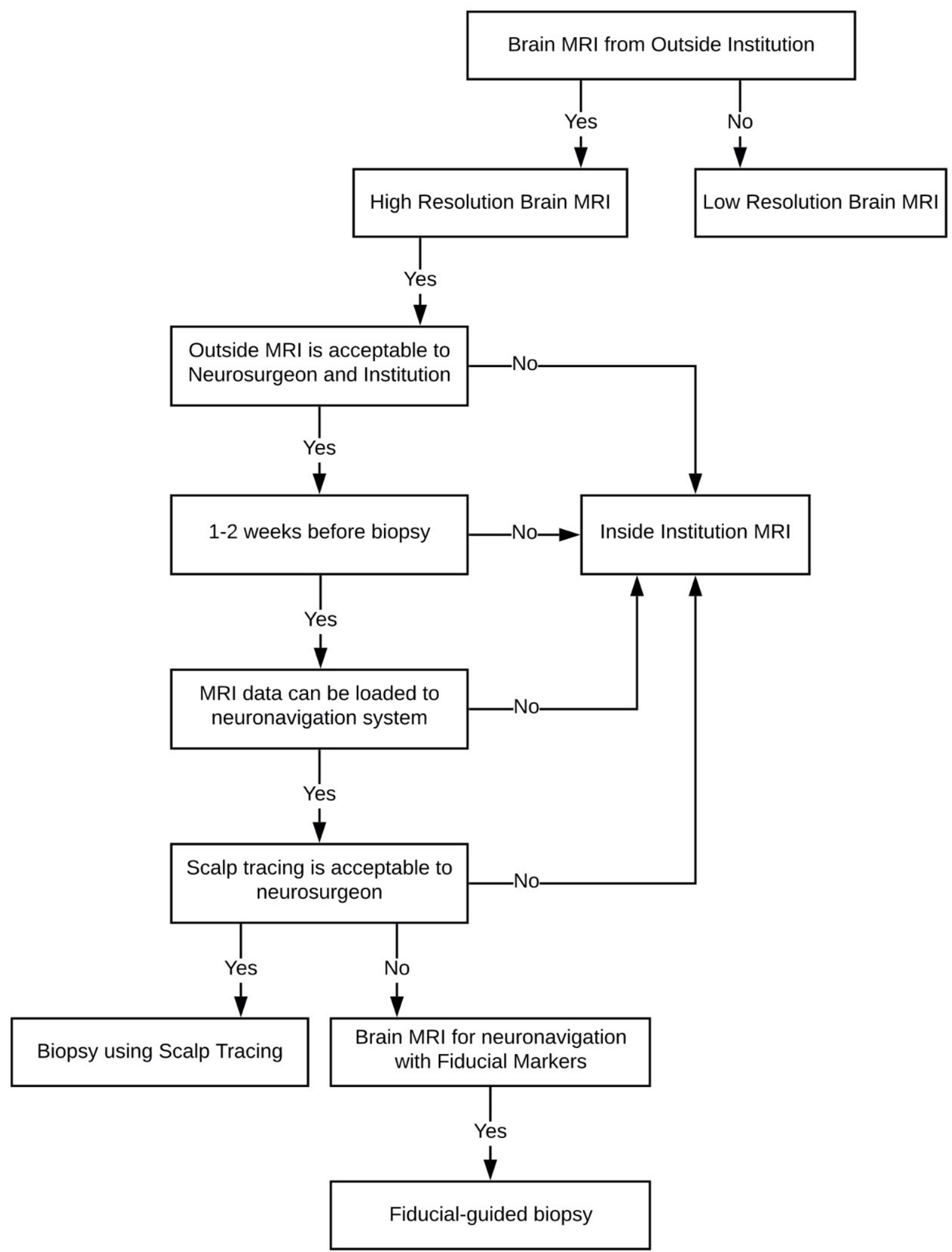

Figure 2: Process flow-chart of clinical workup for patients receiving an MRI scan for stereotactic brain biopsy. 
Citation: FANG FY, POMERANIEC IJ, MASTORAKOS P, et al. (2022) Reducing Institutional Imaging Costs for Stereotactic Brain Biopsy. J Neurosurg Res Rev 5(1):76-83

between diagnosis and surgery, fiducial marker placement, and transferring scans between institutions. Total costs are correlated with the number of MRI scans performed before surgery. The number of scans range depending on their resolution and location at which they are taken.
Table 1 characterizes stepwise costs associated preoperative workup. Total costs for each step depend on percent insurance reimbursement and maximum negotiated charge. Private insurance companies like Aetna reimburse $100 \%$ of hospital charges, whereas Medicare reimburses only $80 \%$ of negotiated charges. As a result, hospitals receive

Table 1: Insurance-specific and hospital-specific associated charges for MRI for stereotactic brain biopsy.

\begin{tabular}{|c|c|c|c|}
\hline \multicolumn{4}{|c|}{ Private Insurance (Aetna) } \\
\hline MRI type & Percent Insurance Reimbursement & Maximum Negotiated Charge & Total Healthcare Costs \\
\hline MRI with contrast (CPT 70552) & $100 \%$ & $\$ 870$ & $\$ 870$ \\
\hline $\begin{array}{l}\text { MRI with and without contrast } \\
\text { (CPT 70553) }\end{array}$ & $100 \%$ & $\$ 990.12$ & $\$ 990.12$ \\
\hline \multicolumn{4}{|c|}{ Public Insurance (Medicare) } \\
\hline MRI type & Percent Insurance Reimbursement & Maximum Negotiated Charge & Total Healthcare Costs \\
\hline MRI with contrast (CPT 70552) & $80 \%$ & $\$ 382$ & $\$ 306$ \\
\hline $\begin{array}{l}\text { MRI with and without contrast } \\
\text { (CPT 70553) }\end{array}$ & $80 \%$ & $\$ 382$ & $\$ 306$ \\
\hline \multicolumn{4}{|c|}{ Other MRI-associated charges } \\
\hline & Transfer fee (CD) & Fiducials (10/patient) & Maximum Associated Charges \\
\hline & $\$ 6.50$ & $\$ 23$ & $\$ 29.50$ \\
\hline
\end{tabular}

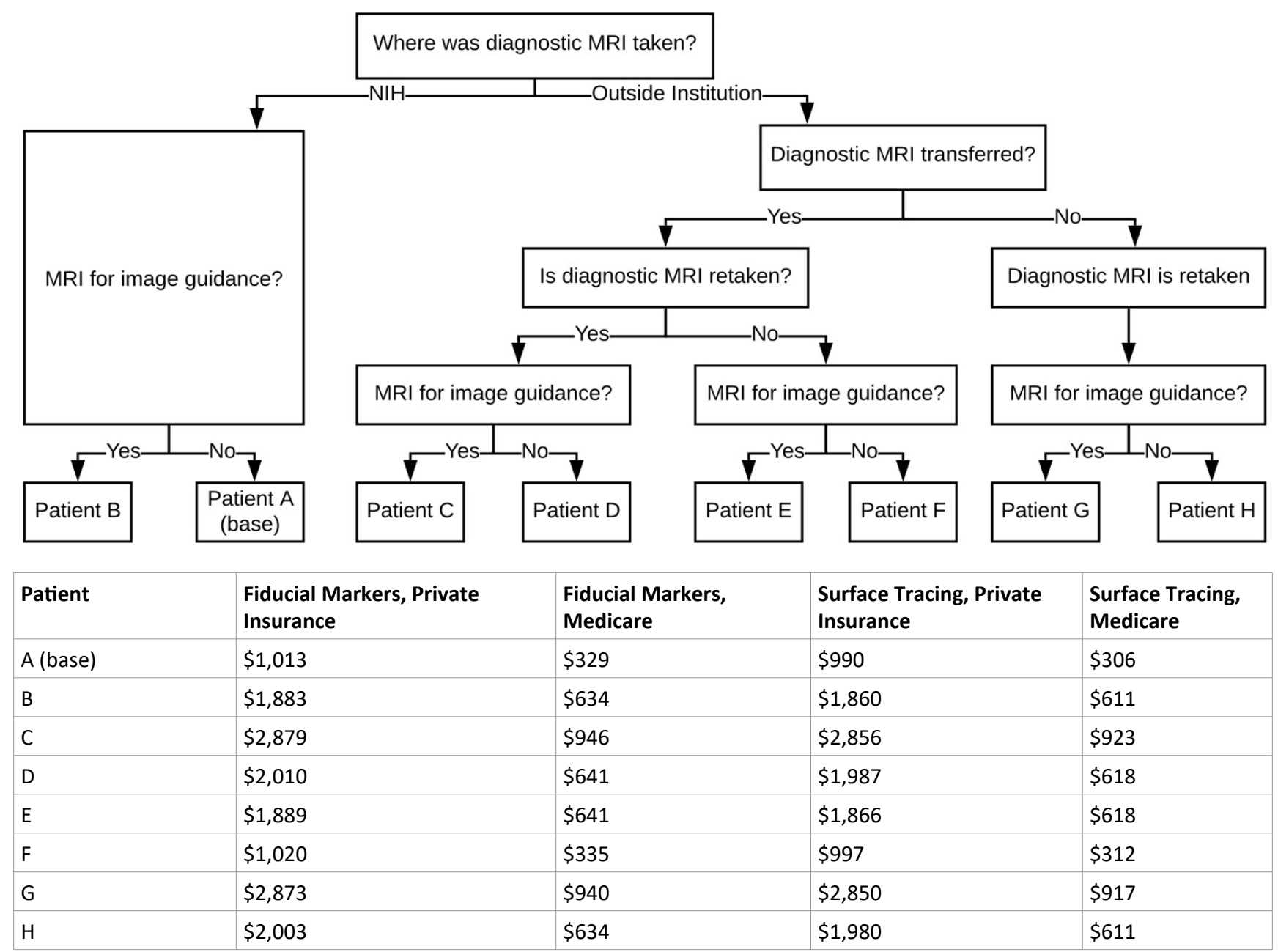

Figure 3: Flow-chart of clinical workup-dependent costs for Patients A-H. 
between $\$ 870-\$ 990$ per patient with private insurance and $\$ 306$ per Medicare patient. Other relevant material costs include transferring scans $(\$ 6.50)$ and placing fiducials (labor not included) prior to MRI scanning (\$23.07).

Figure 3 delineates cost structures of each clinical scenario. All additional patient scenarios are referenced in Appendix A. Three scenarios exemplify different cost drivers and overall costs. Patient A (lowest cost scenario) demonstrates the base scenario with one high-resolution MRI performed at the surgical institution at the time of diagnosis that can be used during surgery. Surface tracing is used for surgical registration. Private insurance cost is $\$ 990$ and Medicare cost is $\$ 306$. Patient $\mathrm{H}$ (intermediate cost scenario) undergoes MRI scanning at an outside institution at the time of diagnosis. This scan is repeated before surgery. Surface tracing is used for surgical registration. Private insurance cost is $\$ 1,980$ and Medicare cost is $\$ 611$. Patient C (highest cost scenario) undergoes MRI scan at an outside institution. However, another MRI is performed either due to extended time between diagnosis and surgery or the need for a higher resolution scan for surgical planning. Fiducial markers are used instead of scalp tracing. Private insurance cost is $\$ 2,879$ and Medicare cost is $\$ 946$.

Comparing the base scenario (Patient $A$ ) with other scenarios (Patient $\mathrm{C}$, Patient $\mathrm{H}$ ) indicates that insurance type and number of additional MRI scans between diagnosis and surgery are predominant cost drivers. Specifically, additional MRI scans performed between diagnosis and surgery contribute $\$ 306$ for Medicare patients versus $\$ 870-\$ 990$ for patients with private insurance. The range of total costs for the aforementioned patient scenarios are $\$ 306-\$ 946$ and $\$ 990-\$ 2,879$ for Medicare and private insurance, respectively. Other factors, such as transfer fee and implementation of fiducial markers, do not significantly impact overall costs.

\section{Discussion}

Stereotactic brain biopsy is required to establish or confirm diagnoses on which comprehensive patient care is based. This essential diagnostic procedure is performed routinely at most medical centers. We questioned if some pre-surgical MRI scans could be avoided without altering the quality of intraoperative neuronavigation.

\section{Savings in context}

Our analysis identified that imaging costs for stereotactic biopsy varied because of the variability in the number of MRI scans performed. For a patient with Medicare, an additional MRI scan costs $\$ 306$, whereas an additional scan for a patient with private insurance costs $\$ 870-\$ 990$. Since other variables such as transfer fees and cost of fiducials do not significantly impact the total cost of an imaging workup, the cost of a scan itself is the major factor contributing to overall cost. As a result, one additional MRI scan effectively doubles the overall cost, and two additional MRI scans effectively triples the overall cost. Eliminating one MRI scan from a two-scan or three-scan workup reduces overall costs by approximately $33 \%$ and $50 \%$, respectively. It is estimated that $57.9 \mathrm{MRI}$ exams were performed per 1,000 inhabitants in hospitals in the United States [14]. In Maryland's population of over 6 million people, if $25 \%$ of all MRI scans are performed on the brain, then there are approximately 86,850 brain MRI scans conducted in the state of Maryland per year [15]. The minimum savings for such a population could be well over $\$ 8.7$ million per year if the number of brain MRI scans were reduced by $33 \%$. While these numbers are estimates, these cost savings represent significant value drivers for healthcare systems that perform thousands of such diagnostic scans. Below we detail different factors that must be considered when determining the need for additional MRI scans.

\section{High-resolution MRI scans}

Imaging costs are reduced by minimizing the number of additional scans. Image guidance during surgery depends on high-resolution 3D MRI. Initial low-resolution diagnostic MRI is unsuitable for neuronavigation and necessitates further high-resolution MRI scanning before surgery. In most cases, initial diagnostic scans from outside institutions are highresolution and suitable if performed within two weeks of surgical biopsy. Ambulatory surgery centers and free-standing radiology clinics often charge less for imaging services than hospitals $[16,17]$. Expanding the role of these institutions in providing imaging studies therefore reduce costs for patients, insurers, and healthcare systems. Higher resolution scans require longer acquisition times, lead to increased patient discomfort and motion artifact, and do not receive higher reimbursement than lower resolution scans [18]. However, obtaining high-resolution rather than low-resolution MRI scans at the outset precludes additional MRI scans without affecting diagnostic biopsy yield.

\section{Timing of surgery}

The time interval between MRI and surgery determines the sufficiency of a single high-resolution scan. If the interval is sufficient to alter the biopsy target or lesion characteristics, then repeat scanning becomes necessary. Sutherland and colleagues reviewed 652 patients with suspected intracranial tumors and found that lesions disappeared between scans, which required cessation of biopsy in five patients [19]. The interval from diagnostic MRI to intraoperative MRIguided biopsy ranged mostly from four to five weeks. New or progressive symptoms or changes to neurologic exam also suggest the need to update scanning. For these reasons, increased time before surgery translates into increased costs. Reducing this interval eliminates additional scans and subsequently overall costs. High-resolution and available initial MRI is suitable for neuronavigation and safe stereotactic brain biopsy, leading to early, definitive diagnosis and treatment. On the other hand, repeating high-resolution MRI scans at the surgical institution or specifically ordering an MRI for neuronavigation introduces unnecessary costs and potentially avoidable delays.

\section{Fiducial markers versus scalp contouring}

Placing fiducial markers requires a dedicated MRI scan at the time of marker placement and increases overall costs. In contrast, scalp contouring performed in real-time in the operating room does not require a dedicated MRI scan. A 
high-resolution MRI scan from any facility can be used to create the 3D imaging model used for neuronavigation.

The clinical advantages of fiducial-guided biopsy compared to contour tracing are controversial. Barnett and colleagues reported $96 \%$ diagnostic success (tissue diagnosis consistent with location and pre-operative MRI characteristics of the biopsied lesion) in 218 patients using image-guided surgical navigation systems with scalp-applied fiducial markers.5 Frameless stereotactic biopsy using fiducial markers demonstrated low rates of morbidity similar to traditional frame-based stereotactic biopsies. Georgiopoulos and colleagues reported similar safety and diagnostic accuracy of frameless surgery compared to frame-based stereotactic biopsy in 28 patients [20]. Amin and colleagues performed stereotactic biopsy in 48 patients using scalp contour tracing without fiducials for registration and reported a $98 \%$ diagnostic success rate with their initial biopsy attempt [21]. Since fiducial and scalp contour methods are both highly accurate, prospective study powered to determine superiority are unlikely to be performed. These studies do not justify the marginal costs and patient inconvenience of fiducial markers. Using scalp tracing instead of fiducial marker placement reduces patient inconvenience, the number of MRI scans, and overall imaging costs required before stereotactic biopsy with comparable quality of care and outcomes.

\section{Private versus public reimbursement}

Traditionally, insurers reimburse institutions set amounts for established procedures. Reimbursement rates, which vary based on patient insurance (private versus national health insurance), represent proportionally the amount hospitals collect for their services. Private insurance options can pay up to the whole amount billed for certain medical procedures. For example, standard Aetna private insurance includes a $\$ 100$ copay and reimbursement of $100 \%$ of billed charges for all brain MRI scans. However, the Centers for Medicare \& Medicaid Services (CMS) maintains negotiating power of these fees with hospitals and provides lower reimbursement compared to private insurance for the same procedures. Medicare reimburses $80 \%$ of the negotiated maximum cost of these procedures [16]. The costs for procedures at some institutions exceed the reimbursement provided by Medicare. In our patient scenarios, the cost differential between private and public reimbursement was approximately $\$ 685$, $\$ 1,300$, and $\$ 1,900$ for one, two, and three brain MRI scans, respectively. These losses are compounded for healthcare systems performing hundreds of stereotactic brain biopsies annually [22].

\section{Healthcare systems and reimbursement}

Costs included in the current analysis represent only one public health system. Since 2014, the state of Maryland has standardized rates for both public and private payers. In return, Maryland hospitals must achieve significant quality improvements such as reducing 30-day readmissions rates and lowering annual Medicare per capital hospital cost growth. These changes will save Medicare over $\$ 330$ million over five years [23]. Overall, this incentive structure yields more coordinated care for patients and encourage prevention and community health planning [24].

Similar approaches can be applied on a micro level to more specialized fields such as neurosurgery. Rather than focusing on costs of individual procedures like MRI or stereotactic biopsy, broader patient plans of care should be considered, examining the entire process of care for redundancy and waste. Physicians ordering MRI scans influence the type and timing of brain imaging which can significantly influence costs and procedural burdens for patients. Neurosurgeons ordering diagnostic imaging before surgery bear an understandably heightened concern with outcome over cost. Despite this, literature has broadly detailed how physicians often neglect the out-of-pocket costs to patients [25] or overall healthcare costs when ordering diagnostic imaging [26]. Knowledge of these costs can reduce the average number of diagnostic [27] and laboratory tests [28]. Some health systems, such as Maryland's unique all-payer system, incentivize physicians to work with community health providers in order to improve patient outcomes, rather than performing diagnostic or treating procedures in a fee for service model. These behaviors can be applied to protocolized preoperative imaging models and reduce the number of MRI scans before surgery without compromising patient care quality.

\section{Value of clinical decisions}

Another key takeaway of procedural costs before surgery relates to value. Although this report details specific costs of procedures for insurers and institutions, healthcare must also fundamentally be considered in terms of value to patients. Value in this system can be defined in terms of meaningful therapeutic value to patients, changes in cost, quality or predictability for health providers, and offsetting risks of payers [29]. Additional MRI scanning does not inherently lead to better outcomes and instead causes inconvenience and wasted time. Since healthcare costs can be highly unpredictable, establishing standard imaging protocols before biopsy reduces this uncertainty for both the patient and the health provider. The goal of providing any clinically meaningful and cost-effective care model rests on understanding underlying costs and benefits of each step along the way. Protocolized medical care accepts necessary procedures and their costs and rejects unnecessary procedures that waste resources.

Frameless stereotactic brain biopsy depends on the digital data of outside MRI scans, neuronavigation models, and patient-specific 3D scalp contouring. The Findable, Accessible, Interoperable, and Reusable (FAIR) Guiding Principles [30] for scientific data management and stewardship aim to extract maximum benefit from research and clinical investments producing digital data. The clinical value of raw MRI data also depends on these underlying principles. Overall, the costs of these imaging processes must be considered systemically and in context, not merely as idiosyncratic and non-generalizable dollar amounts. Understanding how this process fits into a larger system will match the utilization of scarce resources with sophisticated contemporary treatment modalities [3133]. 


\section{Limitations}

This review has several limitations that preclude generalization to other care models. A chief limitation of this review is the lack of patient-specific data and outcomes. Data included in analysis did not include all costs associated with presurgical workup, such as hospital fees, neuroradiologist fees, or MRI suite fees. In addition, the analysis was conducted using public hospital system data from one state (Maryland) and a single private insurance company (Aetna) as a proxy for all private insurance companies. Clinical workup was only considered in the case of stereotactic biopsy for brain tumors. Other pathologies result in different timing of diagnosis and management altogether. Finally, stereotactic biopsy was considered an endpoint in a process to deduce procedural costs. In reality, biopsy should be considered merely a starting point for more definitive and longer-lasting treatments that often draw upon multiple disciplines, including oncology, radiology and neurology. To address these limitations, further inquiry using specific patient data and case series is planned across institutions. This will further clarify tangible costs associated with different processes and understand the relationship between costs and patient outcomes.

\section{Conclusion}

With an increasing shift to cost-conscious resource utilization, particularly during the current COVID-19 pandemic, linking unnecessary procedures to their marginal costs remains an important endeavor. The most significant cost drivers of workup for stereotactic brain biopsy include insurance type and number of additional scans performed between diagnosis and surgery. Cost and waste reductions are achieved through optimizing the timing of high-resolution scans, minimizing the interval between diagnosis and surgery, and disconnecting the placement of fiducial markers with dedicated MRI scans by using the scalp contour method. A cost savings "time-out" when ordering MRI studies before stereotactic brain biopsy reduces unnecessary patient cost and inconvenience. More broadly, modes of healthcare delivery that encourage early and essential procedural steps help to ensure the long-term viability of these practices. These actions can reduce waste without compromising the standard of care for patients with undiagnosed brain lesions.

\section{Conflicts of Interest}

The authors certify that there is no conflict of interest with any financial organization regarding the material discussed in the manuscript.

\section{Authors' Contributions}

Francia Fang and I. Jonathon Pomeraniec have given substantial contributions to the conception or the design of the manuscript, Panos Mastorakos and John D. Heiss to acquisition, analysis and interpretation of the data. All authors have participated to drafting the manuscript, Francia Fang and I. Jonathon Pomeraniec revised it critically. All authors read and approved the final version of the manuscript.

\section{Congresses}

This paper was presented as an e-poster at the 2021 AANS (American Association of Neurological Surgeons) Annual Scientific Meeting that was held in Orlando, Florida, USA on August 21-25, 2021.

\section{Acknowledgements}

The authors would like to acknowledge Ethan Tyler for his medical illustration and Devee Schoenberg for her editing.

\section{References}

1. Samadani U, Stein S, Moonis G, et al. (2006) Stereotactic biopsy of brain stem masses: Decision analysis and literature review. Surg Neurol 66: 484-490.

2. Orringer DA, Golby A, Jolesz F (2012) Neuronavigation in the surgical management of brain tumors: Current and future trends. Expert Rev Med Devices 9: 491-500.

3. Riche M, Amelot A, Peyre M, et al. (2020) Complications after frame-based stereotactic brain biopsy: A systematic review. Neurosurg Rev 44: 301-307.

4. Mendel E, Khoo LT, Go JL, et al. (1999) Intracerebral Whipple's disease diagnosed by stereotactic biopsy: A case report and review of the literature. Neurosurgery. 44: 203-209.

5. Barnett GH, Miller DW, Weisenbeger J (1999) Frameless stereotaxy with scalp-applied fiducial markers for brain biopsy procedures: Experience in 218 cases. J Neurosurg 91: 569-576.

6. Balériaux D, Parizel PM, Matos C, et al. (1993) Stereotactic indications for neuroradiological differential diagnosis. Acta Neurochir (Wien) 124: 31-33.

7. Hartmann M, Heiland S, Sartor K (2002) Funktionelle MRVerfahren in der Diagnostik intraaxialer Hirntumoren: Perfusions- und Diffusions-Bildgebung 174: 955-964.

8. Kickingereder $P$, Willeit $P$, Simon $T$, et al. (2013) Diagnostic value and safety of stereotactic biopsy for brainstem tumors: A systematic review and meta-analysis of 1480 cases. Neurosurgery 72: 873-881.

9. Pinggera D, Kvitsaridtze I, Stockhammer G, et al. (2017) Serious tumor seeding after brainstem biopsy and its treatment-a case report and review of the literature. Acta Neurochir (Wien) 159: 751-754.

10. Tsiouris S, Bougias C, Fotopoulos A (2019) Principles and current trends in the correlative evaluation of glioma with advanced MRI techniques and PET. Hell J Nucl Med 22: 206-219.

11. Wattjes MP, Steenwijk MD, Stangel M (2015) MRI in the diagnosis and monitoring of multiple sclerosis: An update. Clinical Neuroradiology 25: 157-165.

12. Villanueva-Meyer JE, Mabray MC, Cha S (2017) Current clinical brain tumor imaging. Neurosurgery 81 : 397-415.

13. Traboulsee A, Simon JH, Stone L, et al. (2016) Revised recommendations of the consortium of ms centers task force for a standardized mri protocol and clinical guidelines for the diagnosis and follow-up of multiple sclerosis. AJNR Am J Neuroradiol 37: 394-401.

14. (2020) Development OfEC-oa. Magnetic resonance imaging (MRI) exams (indicator). OECD.

15. Rinck PA (2017) Magnetic Resonance in Medicine. (11 $1^{\text {th }}$ edn), The Round Table Foundation, Germany. 
Citation: FANG FY, POMERANIEC IJ, MASTORAKOS P, et al. (2022) Reducing Institutional Imaging Costs for Stereotactic Brain Biopsy. J Neurosurg Res Rev 5(1):76-83

16. (2020) US Centers for Medicare \& Medicaid Services. Procedure Price Lookup.

17. Cleverley J (2017) Identifying the Gap Between Hospital and FreeStanding Prices. Healthcare Financial Management Association.

18. Stucht D, Danishad KA, Schulze P, et al. (2015) Highest Resolution in vivo human brain mri using prospective motion correction. PLoS ONE 10: e0133921-e0133921.

19. Sutherland CS, Kelly JJ, Morrish W, et al. (2010) Identification of disappearing brain lesions with intraoperative magnetic resonance imaging prevents surgery. Neurosurgery 67: 10611065.

20. Georgiopoulos M, Ellul J, Chroni E, et al. (2018) Efficacy, Safety, and duration of a frameless fiducial-less brain biopsy versus frame-based stereotactic biopsy: A prospective randomized study. J Neurol Surg A Cent Eur Neurosurg 79: 31-38.

21. Amin DV, Lozanne K, Parry PV, et al. (2011) Image-guided frameless stereotactic needle biopsy in awake patients without the use of rigid head fixation. J Neurosurg 114: 1414-1420.

22. Morse $S$ (2019) Efficient hospitals operate on $-2 \%$ margins in Medicare payments, MedPAC reports.

23. (2020) US Centers for Medicare \& Medicaid Services. Maryland All-Payer Model.

24. Rajkumar R, Patel A, Murphy K, et al. (2014) Maryland's all-payer approach to delivery-system reform. N Engl J Med 370: 493-495.
25. Pham HH, Alexander GC, O'Malley AS (2007) Physician consideration of patients' out-of-pocket costs in making common clinical decisions. Arch Intern Med 167: 663-668.

26. Rock TA, Xiao R, Fieldston E (2013) General pediatric attending physicians' and residents' knowledge of inpatient hospital finances. Pediatrics 131: 1072-1080.

27. Cummings KM, Frisof KB, Long MJ, et al. (1982) The effects of price information on physicians' test-ordering behavior. Ordering of diagnostic tests. Med Care. 20: 293-301.

28. Feldman LS, Shihab HM, Thiemann D, et al. (2013) Impact of providing fee data on laboratory test ordering: A controlled clinical trial. JAMA Intern Med 173: 903-908.

29. Elton J (2020) What does 'value' mean in healthcare?

30. Wilkinson MD, Dumontier M, Aalbersberg IJ, et al. (2016) The FAIR Guiding principles for scientific data management and stewardship. Scientific Data 3: 160018.

31. (2020) University of Maryland Medical Center (UMMC). Hospital Charges.

32. (2020) Services USCfMM. Procedure Price Lookup Comparison File.

33. Tanaka S, Puffer RC, Hoover JM, et al. (2012) Increased frameless stereotactic accuracy with high-field intraoperative magnetic resonance imaging. Neurosurgery 71: 321-327. 